\title{
A Study of The Peak Boundary Method and Effect Evaluation Based on NaI(TI) Detector for $\gamma$-Spectrum Analysis
}

\author{
Yaozong YANG ${ }^{1}$, Fang FANG $^{1}$, \\ 1. College of Information Science and Technology \\ Chengdu University of Technology \\ Chengdu, China \\ ff@cdut.edu.cn
}

\author{
Jianfeng $\mathrm{HE}^{1,2}$, Junjun RAN ${ }^{1}$ \\ 2. Software College \\ East China University of Technology \\ Nanchang, China \\ hjf_10@yeah.net
}

\begin{abstract}
In order to accurately analyze the result, which comes from quantitative and qualitative detection based on gamma energy spectrum of NaI(T1) detector, peak boundary has become one of the main factors which influence the spectrum analysis. By implementing some common boundary determining algorithms in the Matlab such as simple comparison method, derivative method, symmetry zero-area method, the full width method and gaussian function fitting method, as well as comparing effectiveness among those algorithm, priority of those boundary algorithms is evaluated. At the same time, because the boundary determining algorithm based on traditional gaussian fitting is not ideal, the new boundary determining algorithm based on the least squares fitting of gaussian function with weighting factor $W$ is proposed. The practice verifies that this method is stability and can obtain preferable convergence result in boundary determining of unimodal or combination peaks.
\end{abstract}

Keywords- $\gamma$-spectrum; Gaussian fitting; Weighted least squre fitting; boundary determine

\section{INTRODUCTION}

The ultimate goal of Gamma energy spectrum data processing is qualitative analysis and quantitative analysis. It's an important part of qualitative analysis witch contains searching peaks in $\gamma$-spectrum line, how to calculate the position of every peak in $\gamma$-spectrum line accurately and how to identify whether the sample contains any kinds of nuclide according to the energy of the main peaks and calibration peaks. Peak area calculation is the key technology of quantitative analysis, the calculation of peak area could be affected directly by boundary channel selecting for the characteristic peaks, and even the confirmation of peak position be in the condition with combination line. In addition, as a result of the limited energy resolution of $\mathrm{NaI}(\mathrm{T} 1)$ gamma-ray detectors, the $\gamma$-spectrum is complex with added statistical fluctuation, which adds the difficulties of finding out all peaks accurately. The reliability and result of qualitative analysis is affected directly by the effect of boundary determining, much more researches are necessary to go into the methods of boundary determining for characteristic peaks.

The peak boundary can be determined by the following methods: (1)According to experiment, input the left-right boundary channel directly by observing the $\gamma$-spectrum line, (2)Determine the boundary in the process of peak searching based on the characteristic of methods of peak searching, including Simple comparison method, Derivative method, Symmetric zero area method etc. (3)Determine the boundary channel by Full width method (the boundary channel which point to the $1 / 10$ of the maximum ), (4) Determine the boundary by confidence interval with using Gauss function fitting methods. In this article, the above methods and Matlab simulation experiments to determine the boundary have been described in detail, Gaussian function fitting with a weighting factor $\boldsymbol{W}$ has been realized, and the quality of the boundary have been evaluated by comparison. Determine boundary

\section{DETERMINE BOUNDARY WHILE PEAK SEARCHING}

\section{A. Simple comparison method}

With the use of simple comparison method in peak searching, the left-right peak boundary channel $\mathrm{i}-\mathrm{L}$ and $\mathrm{i}+\mathrm{R}$ can be determined through a comparison discriminant:

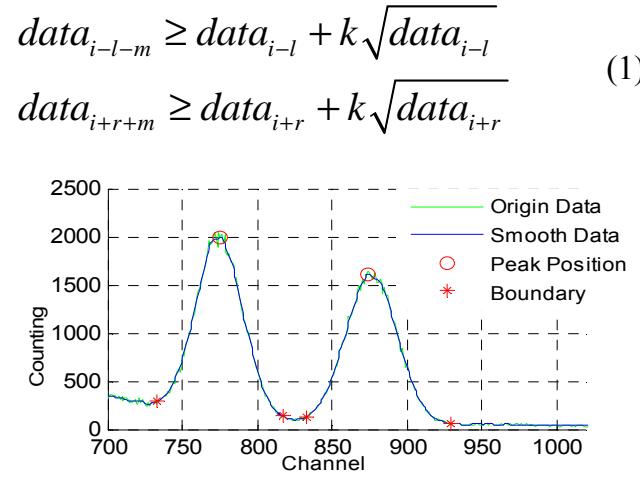

Figure 1. Boundary determined by Single Compare method $\left({ }^{60} \mathrm{Co}\right.$, Channel:700-950)

The parameter $\mathrm{k}$ is a setting threshold value, $\mathrm{m}$ is width factor for peak searching, $l$ and $r$ is the maximum gain channel of left and right, $i$ is the found peak position. It would be recorded as boundary when the points satisfy the discrimination from $i-l$ to $i+r$ channel. One thing to note is that when meeting a trailed peak, the discrimination might not be satisfied, so parameter $l$ and $r$ should be limited to lightly greater than FWHM. Take ${ }^{60} \mathrm{Co} \gamma$-spectrum (Channel: 700-950) in experiment, the result has shown in Figure 1. For the relative separated total-energy peak, simple 
comparison method could get better result, but it can not deal with trailed peak automatically, not to mention the selection of the combination line.

\section{B. Derivative method}

In the mathematical analysis, differential coefficient can reflects the data characteristics of continuous function well, applying Derivative method to this is easily to find peaks and select boundary channel by these characteristics ${ }^{[1]}$. The specific methods is: (1)First derivative method: the zero value point where first derivative changes from positive to negative. (2) Second derivative method: the positive maximum point of second derivative. (3) Third derivative method: the zero value point where third derivative changes from

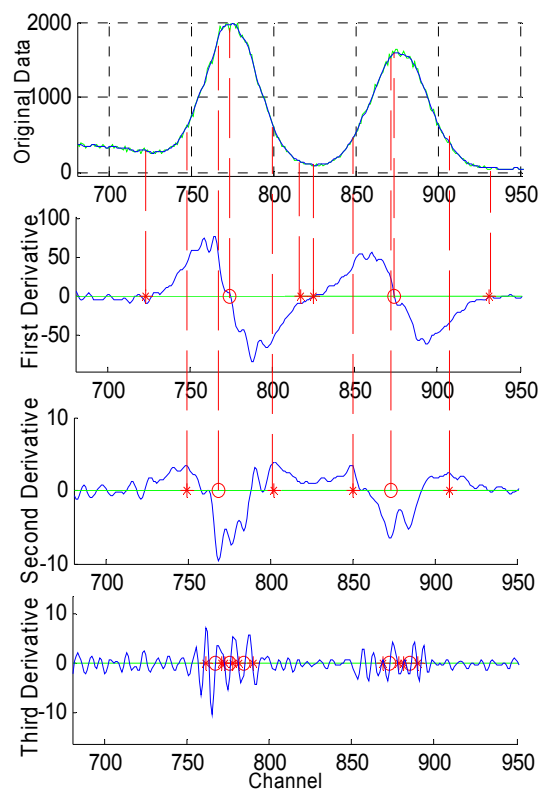

positive to negative.

Figure 2. Boundary determind by Derivative method $\left({ }^{60} \mathrm{Co}\right)$

Make an assay of ${ }^{60} \mathrm{Co}$, as in Figure 2, the results indicate that: (1) The Third derivative method is hardly used in analysis of $\gamma$-spectrum detected by $\mathrm{NaI}(\mathrm{TI})$ detector whose resolving capability is limited because of its too sensitive especially in statistic fluctuation. (2) Compared the corresponding boundary channel selected by first derivative value and the second, it can be found that the peak section ascertained by the second derivative is more narrow and have a higher resolving capability. Moreover, at the inside of gauss peak, effected by the higher counting and statistic fluctuation, boundary difference is more influential on the calculation of peak area, but the peak section is relatively broad and the counting at the boundary channel is low when using first derivative. So the First derivative method is more reasonable for the $\gamma$-spectrum detected by a limited energy resolution $\mathrm{NaI}(\mathrm{TI})$ detector.

\section{Symmetric zero-area method ${ }^{[2]}$}

In order to eliminate the relativity of the sensitivity of peak searching and energy spectrum, standard deviation of the transformation spectrum is always used as unit peak searching, in other words, when the deviation of transformation spectrum and its standard deviation get a positive maximum value and the value exceed a threshold--a fixed number $f$, the value could be regarded as peak. The choice of the boundary: the negative peak position beside positive peak of $S S_{i}$.

$$
S S_{i}=\frac{\bar{y}}{\Delta \bar{y}_{i}}=\frac{\sum_{j=-m}^{m} C_{j} \operatorname{data}_{i+j}}{\left(\sum_{j=-m}^{m} C_{j}^{2} \operatorname{data}_{i+j}\right)^{\frac{1}{2}}}>f
$$

The result of peak searching and boundary determining showed in Figure 3. Instability is shown from the result. The determined left and right boundary of the peak at the 770th channel is better, otherwise, at 870th channel, the left boundary is higher but the right is lower than normal level, result to poor stability. As the erratic fluctuation of negative peak position after calculations, the negative peak may not be ascertained at a fixed point accurately, the boundary channel would be various by different methods.

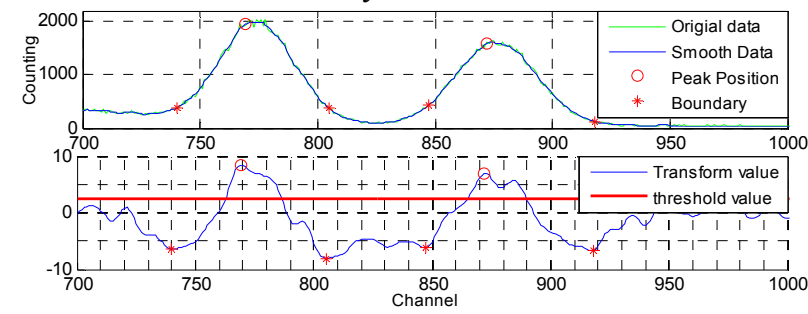

Figure 3. Boundary determind by Symmetric zero-area method $\left({ }^{60} \mathrm{Co}\right)$

\section{DETERMINE BOUNDARY BY FULL WIDTH METHOD}

Using the full width of peak to determine the peak boundary channel is based on the left and right bottom of the peak as full width, making the width at one tenth of the peak maximum as full width, the channel on both sides of the full width is the left and right boundary. And the peak height can be calculated by left and right bottom of the peak. The detail procedure as follows: (1)Select the left and right bottom of peak. Similar to the method of formula (1) showed in searching peak with Simple compare method to find the bottom, here we are looking for the boundary rather than the bottom, the left or right of the boundary may have smaller counting, take left side as an example: a smaller number data point may exist between $i-l-m$ and $i-l$, the bottom is between $i-l-m$ and $i-l$, it is to say that the point hasn't get the bottom. So meliorate formula (1) as follow:

$$
\sum_{j=1}^{m}\left(\operatorname{data}_{(i-l-j)} \geq \operatorname{data}_{i-l}+k \sqrt{\operatorname{data}_{i-l}}\right)==m
$$


The neighbor point would be compared first in formula (4), the bottom channel would be confirmed when continues $\mathrm{m}$ points meet the requirements.

(2) Calculate the peak height $\Delta h: \Delta h=$ data $_{p}-$ data $_{b}$. data $_{p}$ is maximum counting, data ${ }_{b}$ is mean value of left and right bottom channel.

(3) Determine the boundary channel $c h_{L} \& c h_{R}$ at $\left(\frac{1}{10} \Delta h\right)_{L} \&\left(\frac{1}{10} \Delta h\right)_{R}$, respectively.

The effect of determining boundary by Full width method is shown as Figure 4. The peak section is a little narrow and counting in peak section is high, so some of effective counting may lose. However the trailed peak could be dealt well. And Full width method applies to single peak undisturbed but not combined peaks.

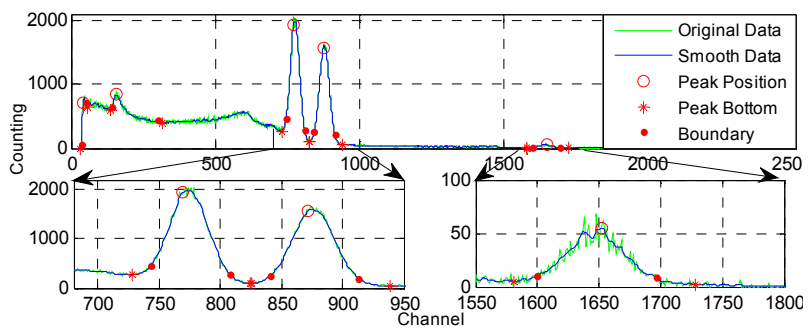

Figure 4. Boundary determined by Full width method. $\left({ }^{60} \mathrm{Co}\right)$

\section{WEIGHTED GAUSSIAN FUNCTION FITTING METHOD}

\section{A. Gauss fitting boundary principle}

Study shows that on one hand, the feature of $\gamma$-spectrum line is in connection with the specific nuclides decay, and on the other hand, it is closely related to the performance of the detector. The luminous efficiency of the scintillator is not absolute same, the counting of photon collection is in connection with the position of flicker, the number of photoelectron is uncertain and the multiplication factor of photomultiplier tube may gets a fluctuation, and the output pulse amplitude of the scintillation detector would fluctuate around a average value. Adding the factors of energy resolution and detection efficiency, the response of $\mathrm{NaI}(\mathrm{T} 1)$ detector for $\gamma$-spectrum impulse approximately obey the Gaussian distribution as formula (5).

$$
f(x)=\frac{1}{\sqrt{2 \pi \sigma}} e^{-(x-\mu)^{2} / 2 \sigma^{2}}
$$

Figure 5 shows that the relationship between FWHM and the variance is FWHM $=2.355 \sigma$. Consider the area of $f(x)$ is 1 , and the corresponding probability of confidence interval $P(\mu-\sigma<X \leq \mu+\sigma)=68.3 \% \quad, P(\mu-2 \sigma<X \leq \mu+2 \sigma)=95.5 \%$ ， $P(\mu-3 \sigma<X \leq \mu+3 \sigma)=99.7 \%$, is shown as Figure 5 .
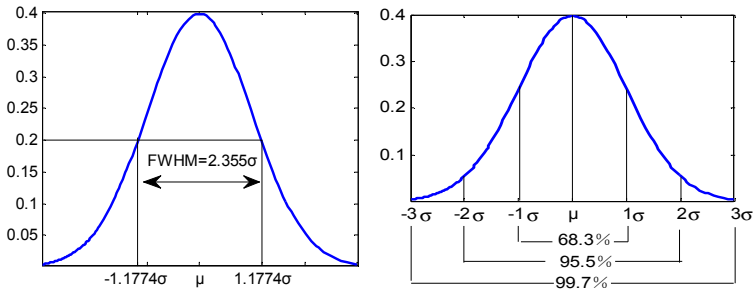

Figure 5. Gaussian Function and Confidence Interval

There isn't a uniform criterion for the selection of confidence probability, so it's enough to get a suitable confidence interval. Experiment have shown that selecting boundary in $\mu \pm 3 \sigma$ confidence interval (99.7\% confidence probability $)^{[3]}$ is not practical because of the peak section is too broad, the $\mu \pm 2.58 \sigma$ confidence interval $(99.0 \%$ confidence probability) is better.

\section{B. Least squares fitting for Gaussian function}

Curve fitting is a process seeking a relatively simple analytic function to approximate a data set[4]. Methods most referenced $[5,6,7]$ on $\gamma$-spectrum data processing is iterative approach, using a large amount of loop iteration which would cost amount computations, make it is hard to be solved with matrix. Considering the unique advantages of MATLAB on matrix operation[8], the least square fitting is adapted to solve a normal equation. In order to improve the fitting effects, a weighting factor is added and the formula of weighted least square fitting is deduced in theoretical process in detail.

The least square fitting is to find a function which could get the minimum of the residual sum of the data set and the result of fitting, that is, minimize $\|y-\hat{y}\|_{2}, y$ is the original data set, $\hat{y}$ is the fitted data set, $y-\hat{y}$ is residual vector, signed by $r$.

Set $\mu=a_{3}, \sigma=\sqrt{1 / 2 a_{2}}$, the equation (5) switch to

$$
a_{1} e^{-a_{2}\left(x-a_{3}\right)^{2}}=y
$$

Taking logarithm on both sides ${ }^{[9]}$ of equation (6): $\ln \left(a_{1}\right)-a_{2}\left(x-a_{3}\right)^{2}=\ln (y)$, or

$$
\ln \left(a_{1}\right)-a_{2}\left(x-a_{3}\right)^{2}=\ln (y)
$$

Set $c_{1}=-a_{2}, c_{2}=2 a_{2} a_{3}, c_{3}=-a_{2} a_{3}+\ln \left(a_{1}\right)$, then the over-determinant equations can be got: $c_{1} x^{2}+c_{2} x+c_{3}=b$, and for data vectors, that is :

$$
A \boldsymbol{c}=\boldsymbol{b}
$$

And in this equation 


$$
\boldsymbol{A}=\left[\begin{array}{lll}
x_{1}^{2} & x_{1} & 1 \\
x_{1}^{2} & x_{1} & 1 \\
\vdots & \vdots & \vdots \\
x_{m}^{2} & x_{m} & 1
\end{array}\right], \quad \boldsymbol{c}=\left[\begin{array}{l}
c_{1} \\
c_{2} \\
c_{3}
\end{array}\right], \quad \boldsymbol{b}=\left[\begin{array}{l}
b_{1} \\
b_{2} \\
\vdots \\
b_{m}
\end{array}\right]
$$

This is over-determinant equations, so it is hardly to find accurate solution. A compromised solution should be found out with least squire method, i.e. a solution could minimize $\|\boldsymbol{r}\|_{2}$. The residual vector could be formulated by: $\boldsymbol{r}=\boldsymbol{b}-\boldsymbol{A c}$.

Set $\rho=\|\boldsymbol{r}\|_{2}^{2}$, then simplify the formula ${ }^{[10]}$, we can get:

$$
\begin{array}{rl}
\rho=\|r\|_{2}^{2}=r^{T} & r=(b-A c)^{T}(b-A c) \\
& =b^{T} b-b^{T} A c-c^{T} A^{T} b+c^{T} A^{T} A c \\
& =b^{T} b-2 b^{T} A c+c^{T} A^{T} A c
\end{array}
$$

In order to minimize $\rho$ with respect to vector $\boldsymbol{C}$, set $\partial \rho / \partial c=0$. With using linear algebra derivation rules, we can get:

$$
\frac{\partial \rho}{\partial \boldsymbol{c}}=-2 A^{T} \boldsymbol{b}+2 A^{T} A \boldsymbol{c}
$$

That is, there will be $-\boldsymbol{A}^{T} \boldsymbol{b}+\boldsymbol{A}^{\boldsymbol{T}} \boldsymbol{A} \boldsymbol{c}=0$ established that the $\boldsymbol{r}$ minimum value should exist, or

$$
\left(A^{T} A\right) c=A^{T} b
$$

Solve equation (11), we can get coefficient vector $\boldsymbol{C}$, then the $\mu$ and $\sigma$ by variable replacement. That is, the least square Gauss fitting is achieved. Experimenting on the total-energy peak of ${ }^{137} \mathrm{Cs} \gamma$-spectrum, the result (background subtracted by linear function) is shown as Figure 6 .

Obviously, the fitting result is disappointed. By analysis the experiment result, the reasons have been found that because of the logarithm transform, the least square fitting get the residual vectors after logarithm transform minimum but not the origin residual vector, that is, $\min \left(\|\boldsymbol{b}-\boldsymbol{A c}\|_{2}^{2}\right)$ after transform does not equal $\min \left(\|\boldsymbol{y}-\hat{\boldsymbol{y}}\|_{2}^{2}\right)$ before transform. Due to the nonlinear properties of logarithm transformation, the data transformed nonlinearly, the larger number reduced more than smaller numbers, so the effects of low counting points were magnified. What people concern more are the high counting points, but, in reality, the larger residual the high counting points get.

In order to solve this problem, introduce a weighting factor $\boldsymbol{W}$ for $\boldsymbol{r}$. The weighting factor should be opposite to the negative trend of logarithm transformation or follow the stream of the original data. Here, take the normalized value of original data as weighting factor:

$$
\boldsymbol{W}=\frac{1}{\sum_{i=1}^{i=n} b_{i}}\left(\begin{array}{lll}
b_{1} & & 0 \\
& \ddots & \\
0 & & b_{n}
\end{array}\right)
$$

The weighted residual vector is $\boldsymbol{r}=\boldsymbol{W}(\boldsymbol{b}-\boldsymbol{A c})$, by the same theoretical derivation above, the normal equation for weighted least square fitting is be deduced:

$$
A^{T} W^{T} W b=A^{T} W^{T} W A c
$$

The result of weighted least square fitting for gauss function is as shown in Figure 6.

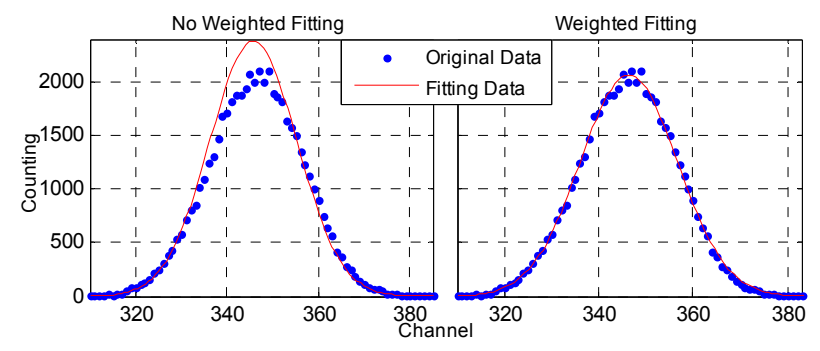

Figure 6. Gaussian Least Square fitting

At last, experiment on ${ }^{137} \mathrm{Cs} \gamma$-spectrum, the result is shown as Figure 7.

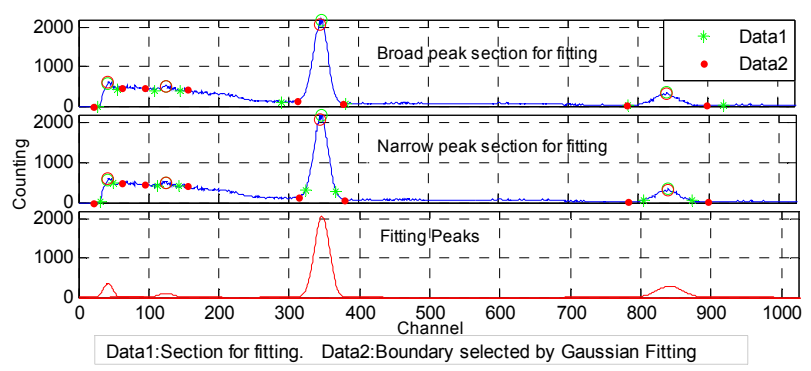

Figure 7. Boundary determined by Weighted Gaussian function fitting method. $\left({ }^{137} \mathrm{Cs}\right)$

Figure 7 shows that no matter broad or narrow peak section to be selected for fitting, weighted Gaussian fitting method get a reasonable boundary and even a certain calibration for peak position. The excellent results and stability have been exhibited in weighted Gaussian fitting method.

\section{CONLISION}

According to the various boundary determining methods above, the boundary have been processed with all the methods by programming with MATLAB and the evaluation of those has been given. The simple compare method is 
sensitive to trailed results, the first derivative method could get a relative better effect and that the second and third are too sensitive to determine the boundary, Symmetric zero area method is unstable, boundary selected by the method is various, although the full-width method could prohibit trailed efficiently, make a narrower peak section, the Gaussian function fitting method costs more computation than others, especially, by introducing weighting factors, the method of weighted Gaussian least square fitting that based on solving normal equations have been suggested, so as to the boundary can be selected accurately and suitably for both single and combination peaks. The weighted Gaussian fitting methods have got a better result in determining boundary for both unimodal peak and combination line.

\section{ACKNOWLEDGMENT}

This work was supported by Natural Science Fundation from Jiangxi Province (N0: 20114BAB211026).

\section{REFERENCES}

[1] Hu Guangchun, Liu Xiaoya, Hao Fanhua. The Analysis of Locating Algorithm for Spectral Peaks of Spectrum. China Nuclear Science and Technology Report, 2008, 02, 1-10
[2] Pang Jufeng, Zheng Guifang, Hou xiaofeng. The symmetric zero-area conversion method for peak search. Atomic Energy Science and Technology. Vol.21, No.3, 1987

[3] Liu Yonggang. Study on $\gamma$-Spectrum Data Analysis Methods. Beijing: China University of Geosciences, 2011

[4] Gerald Recktenwald. Numerical Methods with MATLAB Implementation and Application. Wu Weiguo. Beijing: China Machine Press, 2004

[5] Pang Jufeng. $\gamma$-spectrum data anlysis. Xi'an:Shanxi Science and Technology Press, 1990

[6] Guo Haimin, Dai Jiacai. Cased Hole Formation Parameter Logging. Beijing: Petroleum industry press, 2007

[7] Tian Dongfeng, Gong Jian, Wu Jun, Hu Side. Nuclear material $\gamma$ characteristic spectrum detection and analysis technique. Beijing: Defense Industry Press, 2005

[8] Liu Huanjin, Wang Hui,Li Peng, Liu Yanqi.N Practial Skills of MATLAB. Beijing: Beijing. The Beijing university of aeronautics \&astronautics press, 2011

[9] Zhang Qingxian, Ge Liangquan, Lin Yanchang, et al. The study on spectral shift adjustment and energy resolution monitoring for Gamma-ray fpectrometry. Progress Report on China Nuclear Science \& Technology, 2009, Vol.1, 25-28

[10] Bo Changjiang, Luo Yuesheng. Theory of Matrices. Haerbin. Haerbin Engineering University Press, 2007 